\title{
Spreading of human neutrophils on an ICAM-1-immobilized substrate under shear flow
}

\author{
ZHAN DongYing, ZHANG Yan \& LONG Mian* \\ Key Laboratory of Microgravity, National Microgravity Laboratory and Center of Biomechanics and Bioengineering, Institute of Mechanics, \\ Chinese Academy of Sciences, Beijing 100190, China
}

Received March 18, 2011; accepted June 24, 2011; published online December 27, 2011

\begin{abstract}
Neutrophil (PMN) spreading on endothelium, mediated by the interactions between surface-bound $\beta 2$ integrin and intercellular adhesion molecule-1 (ICAM-1) in the inflammatory cascade, is crucial for PMN post-adhesion and trans-migration in blood flow. The underlying mechanisms by which shear flow regulates PMN spreading dynamics are not well understood. Here, a parallel-plate flow chamber assay was applied to quantify the time course of PMN adhesion and spreading on an ICAM-1-immobilized substrate. Two types of shear flow, steady flows at shear stresses of $0.2,0.5$, and 1 dyne $/ \mathrm{cm}^{2}$ and stepwise flows at 0,1 , and 10 dyne $/ \mathrm{cm}^{2}$, were used to elucidate the impact of shear flow on cell adhesion and spreading. The number of adhered PMNs, the fraction of spreading PMNs and the projected area of spread PMNs were determined and were found to correlate with the distribution of surface-bound $\beta 2$ integrin subunit $(\mathrm{CD} 11 \mathrm{a}, \mathrm{CD} 11 \mathrm{~b}$, or CD18). The results indicate that PMN spreading on an ICAM-1 substrate is bi-directionally regulated under shear flow. CD11a, CD11b and CD18 subunits of $\beta 2$ integrin contribute distinctly to PMN spreading on ICAM-1 substrates. This work provides new insights into understanding PMN spreading on the endothelium, mediated by $\beta 2$ integrin and ICAM-1 under shear flow.
\end{abstract}

neutrophil, spreading, $\beta 2$ integrin, ICAM-1, shear stress

Citation: Zhan D Y, Zhang Y, Long M. Spreading of human neutrophils on an ICAM-1-immobilized substrate under shear flow. Chin Sci Bull, 2012, 57: 769-775, doi: 10.1007/s11434-011-4939-4

Leukocytes are crucial to host defense via extravasation from blood vessels at the site of infection [1]. Neutrophils (PMNs), the largest subpopulation of leukocytes, play a dominant role in acute inflammation [1,2]. In post-capillary venules of inflamed tissue, PMNs attach to the vessel wall through tethering and rolling, followed by firm adhesion and emigration across the endothelium. Tethering and rolling involve the binding of selectins and their ligands, while the subsequent firm adhesion and spreading of leukocytes are mainly mediated by the interaction of integrins and their ligands, where both molecular pairs are expressed, respectively, on leukocytes and endothelial cells [1].

After initial attachment to the endothelium, $\beta 2$ integrins (primarily $\alpha \mathrm{L} \beta 2$ and $\alpha \mathrm{M} \beta 2$ ) expressed on neutrophils contribute to the subsequent adhesion and spreading $[3,4]$.

*Corresponding author (email: mlong@imech.ac.cn) $\alpha \mathrm{L} \beta 2$ (CD11a/CD18 or lymphocyte function-associated (LFA)-1) and $\alpha \mathrm{M} \beta 2$ (CD11b/CD18 or macrophage antigen (Mac)-1) have similar structures, consisting of a common $\beta 2$ chain and a distinct $\alpha$ chain [4,5]. Both receptors specifically recognize their ligand, intercellular adhesive molecule-1 (ICAM-1), which is expressed constitutively on endothelial cells and more highly on inflamed vascular endothelium [4-6]. While all LFA-1 and 5\% of Mac-1 molecules yield a low affinity state on the surface of resting neutrophils [7], their affinity and avidity are remarkably enhanced via conformational change and clustering, resulting in slowed rolling and strengthened adhesion of PMNs [8-10]. Moreover, the remaining $95 \%$ of Mac-1 molecules also rapidly transport from specific granules and secretory vesicles to the cell membrane [7]. Although LFA-1 and Mac-1 work cooperatively to recruit PMNs into the site of inflammation, they may have distinct roles in mediating neutro- 
phil-endothelial interactions, i.e., PMN spreading, particularly at the specific stage of the inflammatory cascade $[11,12]$.

It has been noted that PMN adheres and spreads onto the endothelium under blood flow [13,14]. Mechanical forces regulate leukocyte-endothelium interactions and affect the mechanical properties of leukocytes in the physiological shear stress range $0-30 \mathrm{dyne} / \mathrm{cm}^{2}[3,15,16]$. On the one hand, physiological shear flow facilitates PMN adhesion mediated by selectins, which is a prerequisite for adhesion through integrin $[3,17]$. On the other hand, fluid shear elongates the adhered PMNs and regulates their deformation and spreading dynamics $[18,19]$. It has also been found that PMN spreading is mediated specifically by $\beta 2$ integrin-ICAM-1 interactions, in which the CD11b subunit plays a dominant role [20]. However, the means by which shear flow regulates PMN spreading dynamics mediated by $\beta 2$ integrin-ICAM-1 interactions is not clear. Here, we applied a parallel-plate flow chamber system with welldefined shear stress to mimic the physiological flow in blood vessels. The time course of PMN adhesion and subsequent spreading on an ICAM-1 substrate and the regulation of PMN spreading by $\beta 2$ integrin subunits (CD11a, CD11b, and CD18) were studied.

\section{Materials and methods}

\subsection{Proteins and cells}

Recombinant human ICAM-1 and anti-CD18 monoclonal antibody (mAb) 212701 (mIgG1) were purchased from R\&D Systems (Minneapolis, MN, USA). Anti-CD11a mAb MEM25 (mIgG1) was from Caltag Laboratories (Burlingame, CA, USA) and anti-CD11b mAb 44 (mIgG1) was from Chemicon International (Temecula, CA, USA). FITClabeled goat anti-mouse secondary antibody was obtained from Sigma Co. (St. Louis, MO, USA).

Human neutrophils were isolated from healthy donors using a previously described protocol [21]. Briefly, $4 \mathrm{~mL}$ of anti-coagulant blood was mixed with $4 \mathrm{~mL}$ of $6 \%$ Dextran T-500 (Pharmacia, F1076, Uppsala, Sweden) dissolved in $0.9 \% \mathrm{NaCl}$ solution. Leukocyte-rich and red blood cell-poor plasma was then collected and centrifuged after being layered on lymphocyte separation medium. To remove the remaining red blood cells, the pellet was re-suspended in 9 $\mathrm{mL}$ of pure water for $25 \mathrm{~s}$ and mixed immediately with $1 \mathrm{~mL} 10 \times$ phosphate buffer solution (PBS), and the packed PMNs were suspended in $1 \mathrm{~mL}$ of $\mathrm{HBSS}(-)$ (HyClone, $\mathrm{SH} 30588.01$, without $\mathrm{Ca}^{2+}, \mathrm{Mg}^{2+}$, Logan, UT, USA) containing $0.2 \% \mathrm{HSA}$ and $10 \mathrm{mmol} / \mathrm{L}$ Hepes. Finally, $2 \times 10^{6}-$ $5 \times 10^{6} \mathrm{PMNs}$ were collected and prepared for measurements within $3 \mathrm{~h}$. Prior to the experiments, the PMNs were re-suspended at $0.5 \times 10^{6} / \mathrm{mL}$ in $\mathrm{HBSS}(+)$ (HyClone, $\mathrm{SH} 30268.01$, with $\mathrm{Ca}^{2+}, \mathrm{Mg}^{2+}$ ) containing 0.2\% HSA and $10 \mathrm{mmol} / \mathrm{L}$ Hepes. In some cases, an aliquot of PMNs was fixed using $2 \%$ paraformaldehyde, which served as a control for PMN spreading.

\subsection{Parallel-plate flow chamber assay}

To immobilize ICAM-1 onto the substrate, $1 \mathrm{mg} / \mathrm{mL}$ of ICAM-1 stock solution was diluted to $100 \mu \mathrm{g} / \mathrm{mL}$ with 40 mmol/ $\mathrm{L} \mathrm{NaHCO}_{3}$ (pH 9.0) [22] and then coated on a sterile polystyrene slide inside a region measuring $0.5 \mathrm{~mm} \times 0.5$ $\mathrm{mm}$. After incubation for $1 \mathrm{~h}$ at room temperature, the slide was stored at $4^{\circ} \mathrm{C}$ and washed with $\mathrm{HBSS}(+)$ before use.

A parallel-plate flow chamber was set up by placing the ICAM-1-immobilized slide onto a polycarbonate deck in a chamber with geometry $76 \mathrm{~mm}(L) \times 25 \mathrm{~mm}(W) \times 0.1 \mathrm{~mm}$ $(H)$ (Figure 1(a)). The uniform wall shear stress $(\tau)$ was determined by $\tau=6 \mu Q / W h^{2}$ where $Q$ is the flow rate and $\mu$ is the medium viscosity $(\sim 1 \mathrm{mPa} \mathrm{s})$. The assembled flow chamber was then mounted on the stage of an inverted microscopy (Olympus, IX71, Tokyo, Japan) and the cell suspension was driven by a syringe pump to flow through the

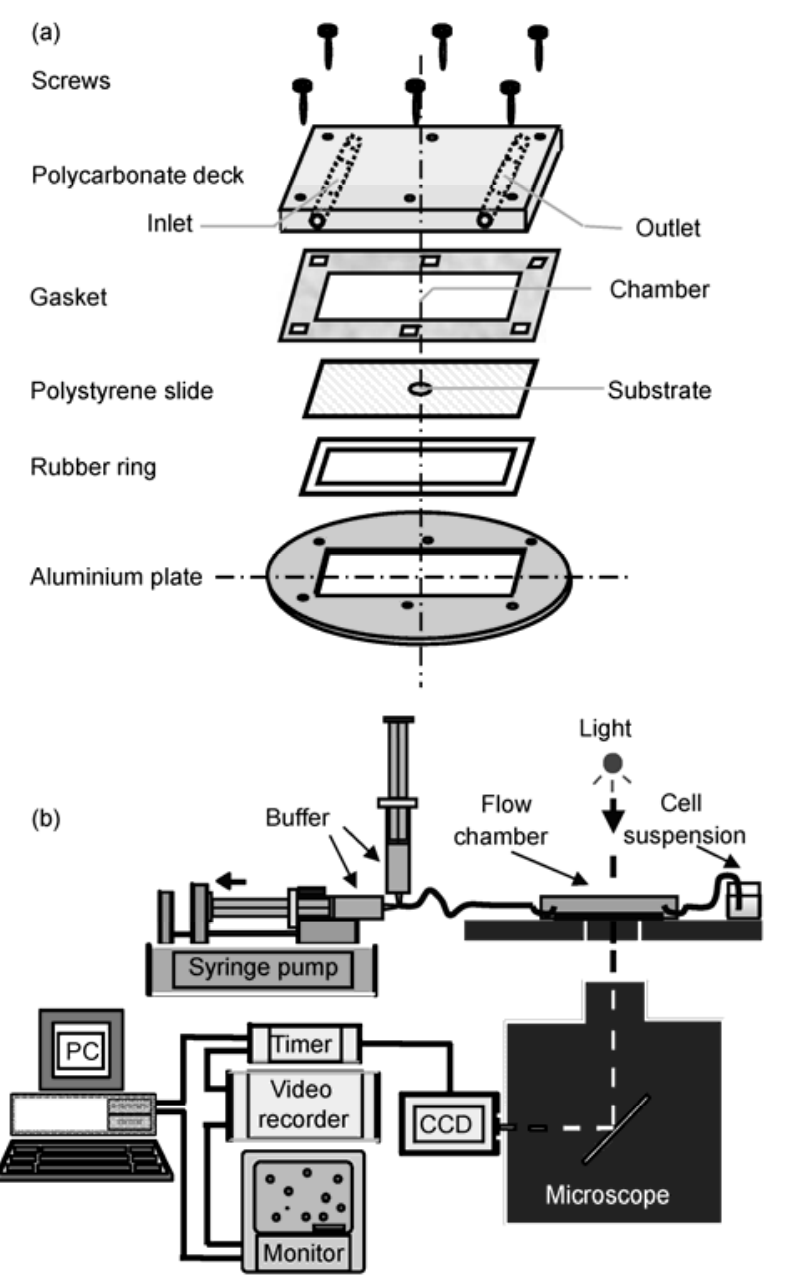

Figure 1 Schematic of a flow chamber assay system. (a) Component assembly of parallel-plate flow chamber. (b) Illustration of parallel-plate flow chamber working system. 
chamber. The adhesion and spreading of PMNs onto the ICAM-1 substrate was observed microscopically inside an area $0.6 \mathrm{~mm} \times 0.45 \mathrm{~mm}$ and the images recorded via a video recorder (Panasonic, AG-4700, Osaka, Japan) were used for off-line analysis (Figure 1(b)).

\subsection{Cell adhesion and spreading measurements}

Two cases of PMN-substrate interaction were defined when PMNs adhered to ICAM-1 substrate. The first was so-called spreading PMN, exhibiting a dark and irregular morphology with a light rim and the second was denoted as nonspreading cells, with round and light morphology (Figure 2(a)) $[20,23,24]$. The fraction of spreading PMN was determined as the ratio of the number of spread PMNs to the total number of adhered PMNs in one frame. Eleven time points in total were tested in each case. All measurements were carried out at least four times. Image J software (NIH, Bethesda, MD, USA) was used to calculate the projected area of the spread PMN.

Two types of shear flow, i.e., a steady flow and a stepwise flow, were used to test PMN adhesion and spreading separately. A steady flow at shear stresses of $0.2,0.5$, or 1 dyne $/ \mathrm{cm}^{2}$ was applied (Figure 2(b)) to study PMN adhesion onto an ICAM-1-immobilized substrate spanning an entire 10-min duration when a PMN appeared in the observation zone. Alternatively, a stepwise flow was used by applying a low flow of 0.2 dyne $/ \mathrm{cm}^{2}$ for the first $1 \mathrm{~min}$ to encourage PMN adhesion onto the ICAM-1 substrate, followed by flows with shear stresses of 0,1 , or $10 \mathrm{dyne} / \mathrm{cm}^{2}$ for the remaining 9 min to test the spreading dynamics of PMNs on the substrate (Figure 2(c)).

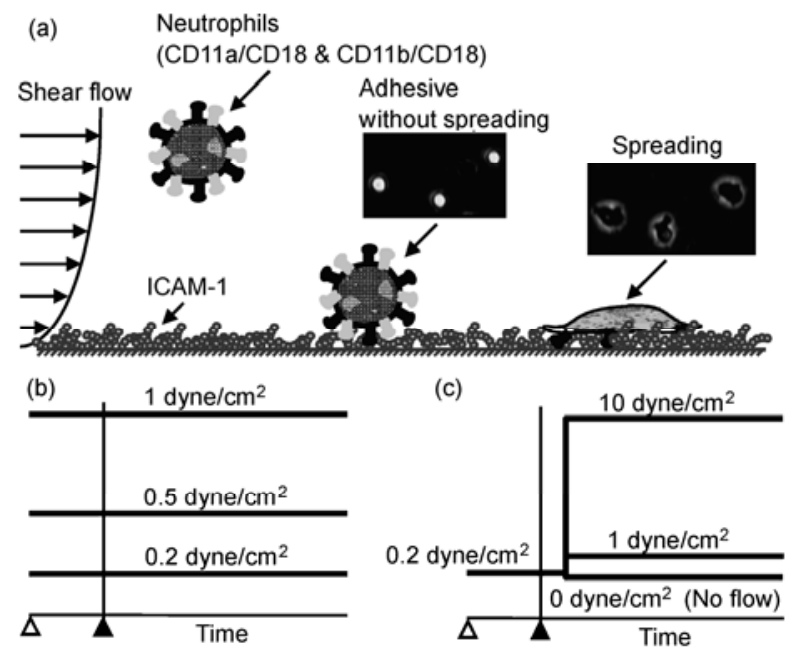

Figure 2 Schematic of human PMNs adhering and spreading onto the ICAM-1 substrate under flow. (a) Illustration of flow chamber measurements. Insert: images showing cell adhesion (middle panel) and spreading (right panel). (b) Steady shear stresses of $0.2,0.5$, and $1 \mathrm{dyne} / \mathrm{cm}^{2}$. (c) Stepwise shear stresses of 0,1 , and $10 \mathrm{dyne} / \mathrm{cm}^{2}$. Symbols $\Delta$ and $\boldsymbol{\Delta}$ denote the start of flow and video timer, respectively.

\subsection{Determination of $\beta 2$ integrin subunit localization}

Localizations of three $\beta 2$ integrin subunits, i.e., CD11a, CD11b, and CD18, constitutively expressed in PMNs, were determined using confocal microscopy (TCS SP2, Leica, Wetzlar, Germany) [25]. Briefly, three aliquots were separated from freshly isolated PMNs and each aliquot was labeled by anti-CD11a (MEM-25), -CD11b (44), and -CD18 (212701) mAbs, respectively. After incubation with FITCconjugated secondary mAbs, collected PMNs were used for PMN adhesion and spreading measurements. The spread cells were fixed immediately after the measurements and the $\beta 2$ integrin subunit localization was then visualized using confocal microscopy. In some cases, PMNs were fixed prior to the measurements and served as a control for nonspreading PMN. Note that only unlabeled subunits contributed to PMN spreading when determining the localization of $\beta 2$ integrin subunits.

\section{Results}

\subsection{Adhesion of PMNs to ICAM-1 substrate is down- regulated by shear stress}

We first tested the adhesion of PMN under a steady flow at shear stresses of $0.2,0.5$, or $1 \mathrm{dyne} / \mathrm{cm}^{2}$ (cf. Figure 2(b)). Under shear flow, the cells appeared to initially settle down and then adhere to the ICAM-1-immobolized substrate. Time courses of PMN adhesion exhibited a transition phase during which adhesion increased with time and a steady phase when it reached equilibrium (Figure 3(a)). It was also found that the critical time point for transition to the steady phase was shortened from $\sim 3 \mathrm{~min}$ at $0.2 \mathrm{dyne} / \mathrm{cm}^{2}$ to $\sim 2$ and $\sim 1 \mathrm{~min}$ at 0.5 and $1 \mathrm{dyne} / \mathrm{cm}^{2}$, respectively. Similarly, the average number of adhered neutrophils at the equilibrium phase was found to inversely correlate with shear stress applied, that is, the value of $177 \pm 20$ cells/frame at 0.2 dyne $/ \mathrm{cm}^{2}$ was 1.5 - and 4.5 -fold higher than those at 0.5 $(122 \pm 25$ cells/frame $)$ and $1(39 \pm 6$ cells/frame $)$ dyne $/ \mathrm{cm}^{2}$, respectively. No PMN adhesion was found when shear stress was above 1 dyne $/ \mathrm{cm}^{2}$ (data not shown). These results indicate that both the transition and steady phases of PMN adhesion depend strongly upon applied shear. Additionally, some of the adhered PMNs were found to spread on the ICAM-1 substrate. The average number of spread neutrophils during the equilibrium phase was also inversely correlated to shear stress for $0.2(91 \pm 8$ cells/frame $), 0.5(73 \pm 14$ cells/frame) and $1(24 \pm 3$ cells/frame $)$ dyne $/ \mathrm{cm}^{2}$ applied (Figure 3(b)).

\subsection{Shear stress modulates PMN spreading on the ICAM-1 substrate}

We further tested the spreading dynamics for adhered PMNs. Here, the fraction of the number of spread cells to the total number of adhered cells was determined frame by 

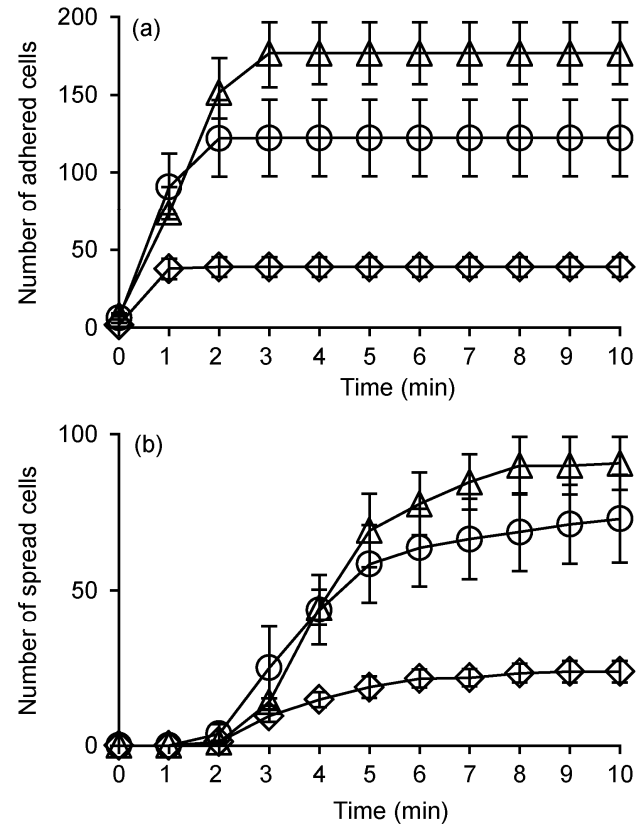

Figure 3 Shear stress dependence of PMN adhesion (a) and spreading (b) on the ICAM substrate at 0.2 (triangles), 0.5 (circles), and 1 (diamonds) dyne $/ \mathrm{cm}^{2}$. Data are presented as mean \pm standard error (SE) of the number of adhered or spread cells/frame from four donors.

frame in each case. Under steady shear stresses of 0.2, 0.5, or 1 dyne $/ \mathrm{cm}^{2}$ (cf. Figure 2(b)), the time course of the spreading fraction appeared to be a sigmoidal curve, where it exhibited a slow phase initially, followed by a phase of rapid increase and reached a steady phase (Figure 4(a)). At each time point, the fraction was slightly enhanced with increasing applied shear stress and reached equilibrium values of $0.54,0.60$, and 0.62 at $0.2,0.5$, and $1 \mathrm{dyne} / \mathrm{cm}^{2}$, respectively. However, no significant differences were found between the values. It was also found that PMN spreading in the absence of shear flow (cf. Figure 2(c)) was dramatically higher (squares in Figure 4(a)) than under shear flow, indicating that low shear inhibited the spreading of adhered PMNs. Note that it was difficult to visualize PMN spreading at high shear stresses because almost all of the cells flowing into the field were carried away when a steady flow corresponding to a shear stress above 1 dyne $/ \mathrm{cm}^{2}$ was applied.

To further test PMN spreading at high shear, a stepwise flow was applied (cf. Figure 2(c)). Here, a low flow of 0.2 dyne $/ \mathrm{cm}^{2}$ was used for the first minute to drive a sufficient number of cells into the field, followed in the subsequent 9 min by a high flow corresponding to 1 or $10 \mathrm{dyne} / \mathrm{cm}^{2}$ to examine the impact of shear flow on PMN spreading. Again, PMN spreading possessed a sigmoidal-like time course at high shears of 1 and 10 dyne $/ \mathrm{cm}^{2}$. The averaged fraction of spreading PMN under a stepwise flow of 1 dyne $/ \mathrm{cm}^{2}$ (diamonds in Figure 4(b); an equilibrium value of 0.77) yielded higher values than those under a steady flow of 1 dyne $/ \mathrm{cm}^{2}$ (diamonds in Figure 4(a)), suggesting that the initial flow pattern is crucial to the spreading of adhered PMNs. Moreover, the stepwise flows applied enabled the cells to continue spreading even at a shear stress as high as 10 dyne $/ \mathrm{cm}^{2}$, and the spreading fraction reached a high equilibrium value of 0.88 (hatched squares in Figure 4(b)). Interestingly, the fraction beyond the 5th min under a 10 dyne $/ \mathrm{cm}^{2}$ shear stress was found to be statistically indistinguishable from those without shear flow (even though the equilibrium value of 0.92 was slightly higher without flow), implying that the shear-induced inhibition in the spreading of adhered PMNs is dramatically abolished at high shear. Overall, the impact of shear flow on PMN spreading was bidirectional, depending mainly on the magnitude of shear flow.

\subsection{Morphology of spread PMN is associated with the action of shear flow}

Spreading of an individual PMN on the ICAM-1-immobilized substrate occurred spontaneously within $1 \mathrm{~min}$. To elucidate the time-lapsed remodeling of cell shape, the projected area for a total of 8-21 cells was measured in each
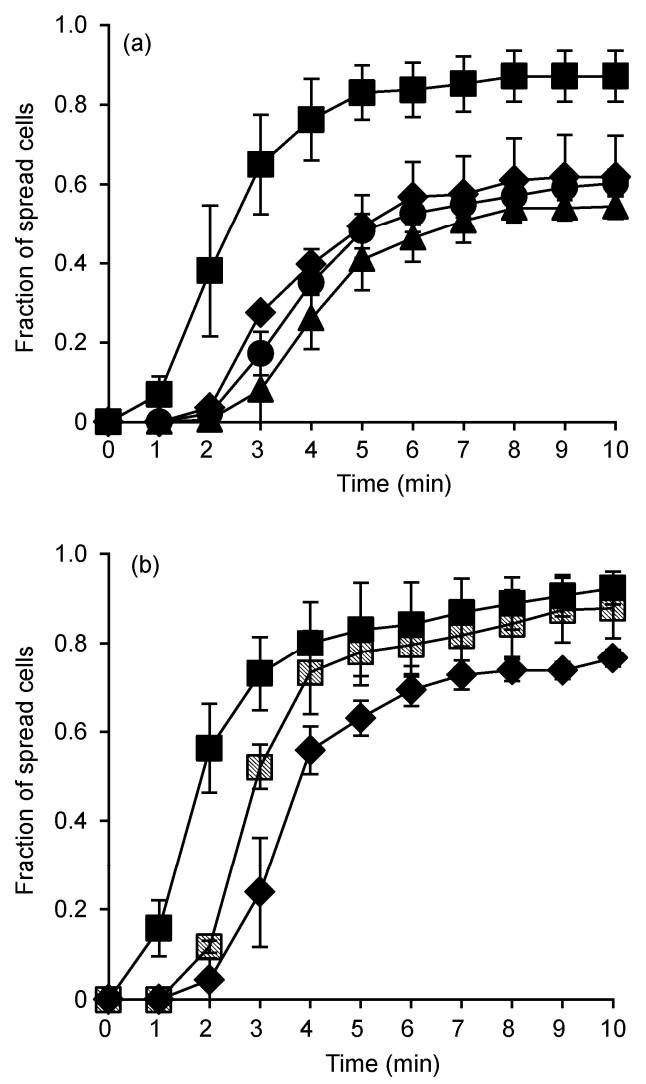

Figure 4 Impact of shear stress on PMN spreading on the ICAM-1 substrate. (a) Time course of PMN spreading fraction at a steady shear of 0.2 (triangles), 0.5 (circles), and 1 (diamonds) dyne/ $\mathrm{cm}^{2}$ (cf. Figure 2(b)) as well as in the absence of shear flow (squares) (cf. Figure 2(c)). Data are presented as mean \pm SE from four donors. (b) Time course of spreading fraction at stepwise shears of 1 (diamonds) and 10 (hatched squares) dyne $/ \mathrm{cm}^{2}$ as well as in the absence of shear flow (solid squares) (cf. Figure 2(c)). Data are presented as mean $\pm \mathrm{SE}$ from four donors. 
case under distinct shear flow. It was found that, under steady flow, the projected areas were similar at 0.2 or 0.5 dyne $/ \mathrm{cm}^{2}$ (275.4 \pm 4.5 and $274.2 \pm 8.1 \mu \mathrm{m}^{2}$, respectively) but lower than those at $1 \mathrm{dyne} / \mathrm{cm}^{2}\left(333.2 \pm 5.8 \mu^{2}\right)$ or without shear flow $\left(312 \pm 7.3 \mu^{2}\right.$ ) (Figure 5(a)). This implies that continuous flow inhibited PMN spreading at low shear but promoted it at high shear. In contrast, under stepwise flow, no significant differences in PMN projected area were observed at $1\left(354.6 \pm 17.8 \mu^{2}\right), 10\left(377.1 \pm 13.9 \mu \mathrm{m}^{2}\right)$ dyne/ $\mathrm{cm}^{2}$ or in the absence of shear flow $\left(329.4 \pm 12.7 \mu \mathrm{m}^{2}\right)$ (Figure 5(b)), suggesting that the shape of firmly-adhered PMNs was stable under the action of stepwise flow. Together, these results indicate that PMN morphology varies slightly with the applied shear flow.

\section{$2.4 \quad \beta 2$ integrin subunits contribute to PMN spreading on the ICAM-1-immobilized substrate}

To elucidate the contribution of $\beta 2$ integrin subunits to PMN spreading on the ICAM-1 substrate, a confocal microscopy was used to visualize the localization of FITClabelled-CD11a, -CD11b, or -CD18 on the cell surface (Figure 6). Three tests of non-spreading PMN (fixed cells; Figure 6(a)), spreading statically (Figure 6(b)) or spreading at a stepwise shear of $10 \mathrm{dyne} / \mathrm{cm}^{2}$ (Figure 6(c)) were conducted. It was found that non-spread PMNs retained a round shape, with a relatively uniform distribution of CD11a,
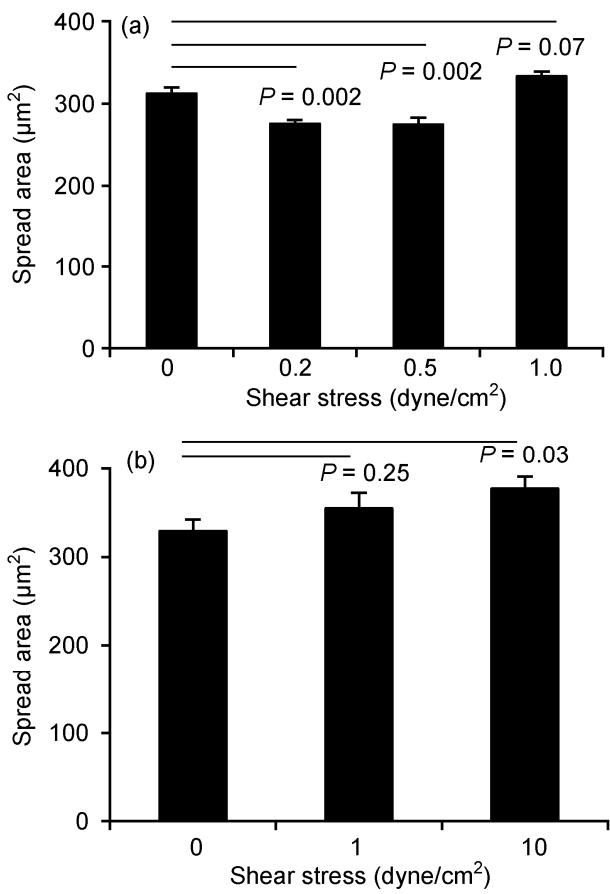

Figure 5 Shear stress dependence of the projected area of spread PMN. (a) Comparison of projected area at steady shears of $0,0.2,0.5$, and 1 dyne $/ \mathrm{cm}^{2}$. Data were obtained from an ensemble of 18-21 cells in each case and are presented as mean \pm SE. (b) Comparison of projected area at steady shears of 0,1 , and $10 \mathrm{dyne} / \mathrm{cm}^{2}$. Data were obtained from an ensemble of 8-16 cells in each case and are presented as mean \pm SE.

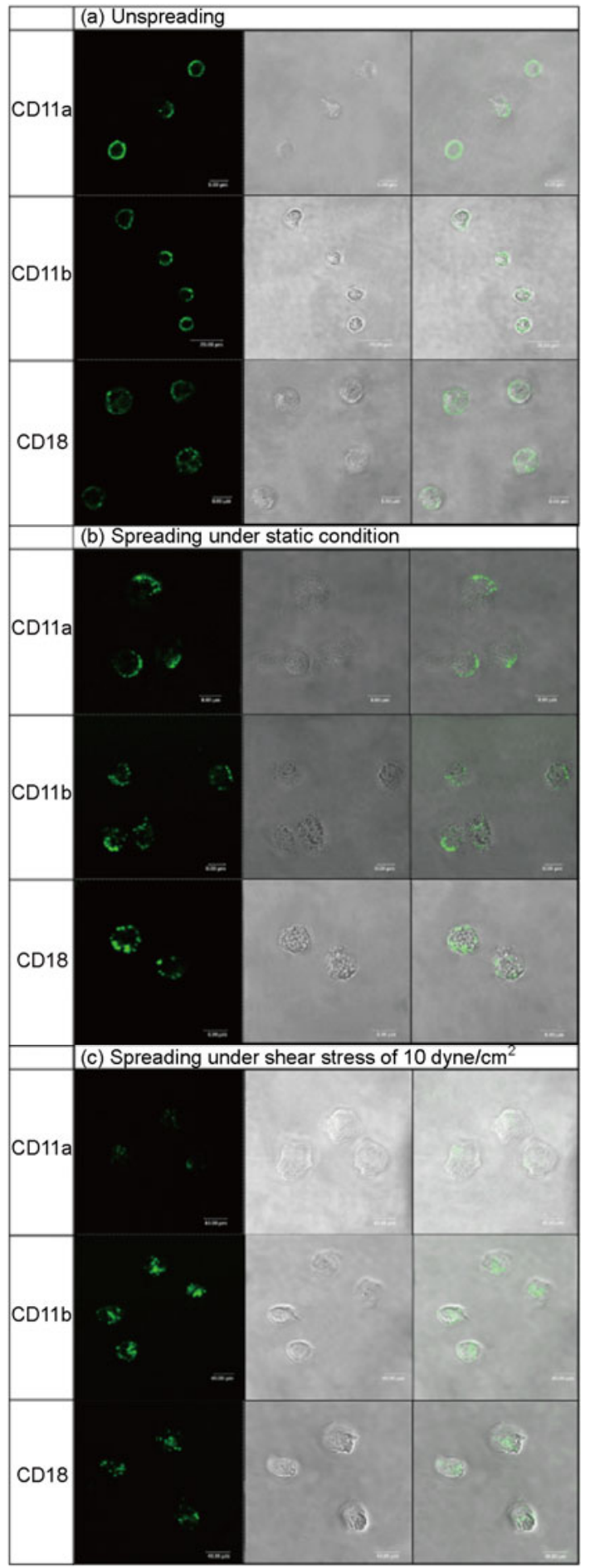

Figure 6 Distribution of CD11a, CD11b and CD18 subunits on the PMN surface for non-spreading (a), spreading statically (b), or spreading under a stepwise shear of $10 \mathrm{dyne} / \mathrm{cm}^{2}$ (c). The 1 st and 2 nd columns show the images from confocal and bright field (BF) microscopy, respectively, while the 3 rd column illustrates the merged images.

CD11b and CD18 on the membrane (Figure 6(a)). In spontaneous spreading at $0 \mathrm{dyne} / \mathrm{cm}^{2}$, the CD11a subunit tended to cluster and polarize towards one region of the PMN membrane, while CD11b and CD18 subunits appeared to 
distribute along the membrane and form aggregates (Figure 6(b)). This specific distribution was also observed when a stepwise flow was applied: the expression of the CD11a subunit was significantly reduced and strong aggregation of CD11b and CD18 subunits were found to cluster in the vicinity of the cell nucleus (Figure 6(c)). These results imply that the three subunits of $\beta 2$ integrin may play distinct roles in PMN spreading under different shear flows.

\section{Discussion}

The goal of the current work was to understand the impact of shear flow on neutrophil adhesion and spreading, because blood flow inevitably influences the adhesion and subsequent spreading behavior $[14,26]$. Two types of flow pattern, a steady flow and a stepwise flow, were used in a parallel-plate flow chamber assay to elucidate PMN adhesion and spreading on an ICAM-1-immobilized substrate. The former flow type was designed to mimic physiological blood flow and the latter type was to isolate the impact on firmly-adhered PMNs. A high ICAM-1 concentration of $100 \mu \mathrm{g} / \mathrm{mL}$ was employed to exclude non-specific interactions introduced by the polystyrene substrate and to ensure that initiation of PMN adhesion and spreading were mainly mediated by $\beta 2$ integrin-ICAM-1 interactions [27]. Three parameters, the number of cells adhering, the fraction of spreading cells, and the projected area of the spread cells, were employed to quantify the impact of shear flow. The results not only quantified the dynamics of PMN adhesion and spreading (Figures 3-5) but also provided insights for understanding the function of $\beta 2$ integrin subunits (CD11a, CD11b and CD18) (Figure 6). The novelty of the current work is the identification of the distinct roles of low or high shear flow on PMN adhesion and spreading (Figures 4 and 5 ). The results are expected to further the understanding of human PMN adhesion and spreading under conditions mimicking physiological shear flow.

It has long been noted that PMNs spread actively on an ICAM-1-immobilized substrate under static conditions [9]. In the current work, an applied shear flow reduced the fraction of spreading PMNs and the projected area of spread PMNs, particularly at steady, low shears of 0.2 and 0.5 dyne $/ \mathrm{cm}^{2}$ (Figures 4(a) and 5(a)). This suggests that shear flow exerted on PMNs inhibits their active spreading onto ICAM substrates. These results are in agreement with the previous observation that PMNs were able to retract their pseudopods when exposed to physiological shear stress $(\sim 1$ dyne $\left./ \mathrm{cm}^{2}\right)[28,29]$. It was also found that the fraction of spreading PMNs was unexpectedly enhanced and the projected area of spread PMNs increased slightly at a high stepwise flow corresponding to a shear of $10 \mathrm{dyne} / \mathrm{cm}^{2}$ (Figure 5), indicating that shear-induced spreading is complex [30], presumably because of the bi-directional function of shear flow.
Interactions involving $\beta 2$ integrin-ICAM-1 binding are assumed to be the major mediators for PMN spreading on ICAM-1-immobilized substrates. It has been reported that the three subunits CD11a, CD11b, and CD18 behave distinctly and yield different dynamics in the mediation of PMN spreading [20]. While all three subunits were uniformly distributed on the PMN membrane before cell spreading, the CD11a subunit tended to translocate to the specific region of the spread membrane during active spreading in the absence of shear flow. In contrast, the CD11b and CD18 subunits were mainly distributed at the center of the cell to inhibit shear-induced passive spreading when a shear flow was applied (Figure 6). Although the $\mathrm{CD} 11 \mathrm{~b} / \mathrm{CD} 18$ molecules are proposed to play the dominant roles inhibiting shear-induced spreading, the underlying mechanism of active or passive spreading requires investigation in future studies.

The authors are grateful to Dr. Quhuan Li of the South China University of Technology for technical assistance and helpful discussions. This work was supported by the National Natural Science Foundation of China (30730032 and 10902117), Chinese Academy of Sciences Knowledge Innovation Project (KJCX2-YW-LO8 and Y2010030), and the National Basic Research Program of China (2011CB710904).

1 Osborn L. Leukocyte adhesion to endothelium in inflammation. Cell, 1990, 62: 3-6

2 Witko-Sarsat V, Rieu P, Descamps-Latscha B, et al. Neutrophils: Molecules, functions and pathophysiological aspects. Lab Invest, 2000, 80: 617-653

3 Lawrence M B, Springer T A. Leukocytes roll on a selectin at physiologic flow rates: Distinction from and prerequisite for adhesion through integrins. Cell, 1991, 65: 859-873

4 Hogg N. The leukocyte integrins. Immunol Today, 1989, 10: 111114

5 Gahmberg C G. Leukocyte adhesion: CD11/CD18 integrins and intercellular adhesion molecules. Curr Opin Cell Biol, 1997, 9: 643650

6 Jun C D, Carman C V, Redick S D, et al. Ultrastructure and function of dimeric, soluble intercellular adhesion molecule-1 (ICAM-1). J Biol Chem, 2001, 276: 29019-29027

7 Sengelov H, Kjeldsen L, Diamond M S, et al. Subcellular localization and dynamics of Mac-1 $(\alpha \mathrm{m} \beta 2)$ in human neutrophils. J Clin Invest, 1993, 92: 1467-1476

8 Stewart M, Hogg N. Regulation of leukocyte integrin function: Affinity vs. avidity. J Cell Biochem, 1996, 61: 554-561

9 Green C E, Schaff U Y, Sarantos M R, et al. Dynamic shifts in LFA-1 affinity regulate neutrophil rolling, arrest, and transmigration on inflamed endothelium. Blood, 2006, 107: 2101-2111

10 Kim M, Carman C V, Yang W, et al. The primacy of affinity over clustering in regulation of adhesiveness of the integrin $\alpha \mathrm{L} \beta 2$. J Cell Biol, 2004, 167: 1241-1253

11 Ding Z M, Babensee J E, Simon S I, et al. Relative contribution of LFA-1 and Mac-1 to neutrophil adhesion and migration. J Immunol, 1999, 163: 5029-5038

12 Ley K, ed. Adhesion Molecules: Function and Inhibition. Basel, Switzerland: Birkhauser Verlag AG, 2007

13 Konstantopoulos K, Kukreti S, McIntire L V. Biomechanics of cell interactions in shear fields. Adv Drug Deliv Rev, 1998, 33: 141-164

14 Simon S I, Green C E. Molecular mechanics and dynamics of leukocyte recruitment during inflammation. Annu Rev Biomed Eng, 2005, 7: 151-185

15 Cao J, Usami S, Dong C. Development of a side-view chamber for 
studying cell-surface adhesion under flow conditions. Ann Biomed Eng, 1997, 25: 573-580

16 Kitayama J, Fuhlbrigge R C, Puri K D, et al. P-selectin, L-selectin, and $\alpha 4$ integrin have distinct roles in eosinophil tethering and arrest on vascular endothelial cells under physiological flow conditions. J Immunol, 1997, 159: 3929-3939

17 Yago T, Zarnitsyna V I, Klopocki A G, et al. Transport governs flowenhanced cell tethering through L-selectin at threshold shear. Biophys J, 2007, 92: 330-342

18 Cao J, Donell B, Deaver D R, et al. In vitro side-view imaging technique and analysis of human T-leukemic cell adhesion to ICAM-1 in shear flow. Microvasc Res, 1998, 55: 124-137

19 Lei X, Lawrence M B, Dong C. Influence of cell deformation on leukocyte rolling adhesion in shear flow. J Biomech Eng, 1999, 121: 636-643

20 Zhan D Y, Zhang Y, Long M. Human neutrophils spreading statically on ICAM-1 substrate mediated by $\beta 2$ integrin (in Chinese). J Med Biomech, 2011 (in press)

21 Alon R, Fuhlbrigge R C, Finger E B, et al. Interactions through L-selectin between leukocytes and adherent leukocytes nucleate rolling adhesions on selectins and VCAM-1 in shear flow. J Cell Biol, 1996, 135: 849-865

22 Sigal A, Bleijs D A, Grabovsky V, et al. The LFA-1 integrin supports rolling adhesions on ICAM-1 under physiological shear flow in a permissive cellular environment. J Immunol, 2000, 165: 442-452

23 Rogers S D, Bignold L P. Which method for the assessment of spreading of polymorphonuclear leukocytes? J Immunol Methods, 1990, 135: 281-284

24 Suzuki K, Namiki H. Cytoplasmic pH-dependent spreading of polymorphonuclear leukocytes: Regulation by $\mathrm{pH}$ of PKC subcellular distribution and F-actin assembly. Cell Biol Int, 2007, 31: 279-288

25 Fukuda S, Schmid-Schonbein G W. Regulation of CD18 expression on neutrophils in response to fluid shear stress. Proc Natl Acad Sci USA, 2003, 100: 13152-13157

26 Konstantopoulos K, Neelamegham S, Burns A R, et al. Venous levels of shear support neutrophil-platelet adhesion and neutrophil aggregation in blood via P-selectin and $\beta 2$-integrin. Circulation, 1998, 98: 873-882

27 Labrador V, Riha P, Muller S, et al. The strength of integrin binding between neutrophils and endothelial cells. Eur Biophys J, 2003, 32: 684-688

28 Fukuda S, Yasu T, Predescu D N, et al. Mechanisms for regulation of fluid shear stress response in circulating leukocytes. Circ Res, 2000, 86: E13-E18

29 Moazzam F, DeLano F A, Zweifach B W, et al. The leukocyte response to fluid stress. Proc Natl Acad Sci USA, 1997, 94: 5338-5343

30 Dong C, Lei X X. Biomechanics of cell rolling: Shear flow, cellsurface adhesion, and cell deformability. J Biomech, 2000, 33: 35-43

Open Access This article is distributed under the terms of the Creative Commons Attribution License which permits any use, distribution, and reproduction in any medium, provided the original author(s) and source are credited. 